\title{
Management of tuberculosis in Wales: 1986-92
}

Verghese Mathew, Mazin Alfaham, Meirion R Evans, Haydn Adams, Roger Verrier Jones, Ian Campbell, Tony Jenkins

\begin{abstract}
Objectives-To describe the epidemiology of childhood tuberculosis in Wales and to assess the standard of management of patients with tuberculosis.

Design-Retrospective study of data retrieved from case notes and review of radiographs of all identified patients.

Subjects-Forty eight cases of tuberculosis and 10 of tuberculosis chemoprophylaxis in children under 15 years of age, in Wales, between January 1986 and December 1992.

Main outcome measures-Management of childhood tuberculosis in Wales compared with the published recommendations of the Joint Tuberculosis Committee of the British Thoracic Society.

Results-Documentation was poor in most of the 48 cases of tuberculosis and only $31(65 \%)$ were formally notified. One third of patients were asymptomatic and were detected by contact tracing; only eight $(\mathbf{1 7 \%})$ were culture positive. Only $20 \%$ of patients from ethnic minorities had previously been immunised with BCG. Management and chemotherapy varied widely. Few patients were managed jointly by paediatricians and chest doctors. Only $10 \%$ completed treatment with a recommended chemotherapy regimen. In $37 \%$ of patients treatment was inadequate, and in the remainder either the choice of drugs or the duration of treatment was inappropriate. No patient died, nor had any relapsed by June 1995 .
\end{abstract}

Conclusions-Very few cases of childhood tuberculosis were managed according to the recommendations of the British Thoracic Society.

(Arch Dis Child 1998;78:349-353)

Keywords: patient management; drug regimens; treatment recommendations

Health Medicine,

South Glamorgan

Health Authority,

Cardiff

M R Evans

Public Health

Laboratory Service

Mycobacterium

Reference Laboratory,

Cardiff Public Health

Laboratory

T Jenkins

Correspondence to:

Dr M Alfaham, Department of Child Health, Llandough Hospital and Community NHS Trust, Penlan Road, Penarth, Vale of Glamorgan CF64 2XX

Accepted 22 December 1997 increase has been in children, although rates for respiratory disease in children have continued to decrease. ${ }^{4}$ Much of the increase has been in areas of urban deprivation, particularly among new immigrants and refugees. ${ }^{45} \mathrm{~A}$ study from inner London confirms that childhood tuberculosis remains an important problem and emphasises the importance of diagnostic vigilance. ${ }^{6}$ A similar resurgence in tuberculosis among children has also occurred in the USA. ${ }^{78}$

The Medical Research Council survey in 1983 found considerable variations in chemotherapy regimens used in children. ${ }^{9}$ In 1990, the Joint Tuberculosis Committee of the British Thoracic Society published recommendations on the chemotherapy and management of tuberculosis in adults and children. ${ }^{10}$ Relatively little is known, however, about how well childhood tuberculosis is managed in current UK clinical practice. This study was undertaken to describe the epidemiology of childhood tuberculosis in Wales and to compare practice with British Thoracic Society management recommendations.

\section{Patients and methods}

Children (less than 15 years of age) in Wales with tuberculosis who were diagnosed between 1986 and 1992 inclusive were identified retrospectively from statutory notifications, laboratory data held by the Mycobacterium Reference Unit of the Public Health Laboratory Service, Cardiff, and by written inquiry to all paediatricians and chest doctors in Wales. After obtaining written consent from the relevant consultant, patient records and radiographs were retrieved and a proforma completed for each patient. Data were sought on: age at diagnosis; sex; ethnic origin; mode of presentation; basis of diagnosis; sputum smear and tissue culture results; BCG immunisation status; and details of treatment received. Presenting radiographs were reviewed by a consultant radiologist (HA). Abnormal chest radiographs were classified as (a) cavitary tuberculosis, (b) noncavitary consistent with tuberculosis, (c) noncavitary unusual for tuberculosis, and (d) other abnormalities. Chest radiographs showing single or multiple cavities with patchy or nodular infiltrates, with or without calcification, were classed as cavitary tuberculosis. Opacities (linear or confluent homogenous shadowing, or both), mainly in the upper zone, with or without calcification, hilar lymph nodes, and/or pleural effusion were classed as non-cavitary consistent with tuberculosis. Characteristics and distributions of shadowing other than those described (for example, lobar consolidation) were grouped as non-cavitary unusual for tuberculosis. All other abnormalities on the chest radiograph (for example, mediastinal widening) were classed as other. 
Table 1 British Thoracic Society recommendations for treatment of childhood tuberculosis and tuberculosis chemoprophylaxis

\begin{tabular}{ll}
\hline Indication & Drug regimen \\
\hline Pulmonary tuberculosis & $\begin{array}{l}\text { (a) Rifampicin, isoniazid, and pyrazinamide for 2 months } \\
\text { followed by rifampicin and isoniazid for } 4 \text { months or } \\
\text { (b) Rifampicin and isoniazid for 9 months }\end{array}$ \\
$\begin{array}{c}\text { Non-pulmonary tuberculosis } \\
\text { Lymph node or bone/joint }\end{array}$ & $\begin{array}{l}\text { Rifampicin, isoniazid, and pyrazinamide for 2 months } \\
\text { followed by rifampicin and isoniazid for 4 months } \\
\text { Rifampicin, isoniazid, and pyrazinamide for 2 months } \\
\text { followed by rifampicin and isoniazid for 10 months } \\
\text { Reningitis }\end{array}$ \\
Other sites & months followed by rifampicin and isoniazid for 4 months \\
Tuberculosis & (a) Isoniazid for 6 months or \\
chemoprophylaxis & (b) Rifampicin and isoniazid for 3 months
\end{tabular}

Patients were classified as confirmed, probable, or possible tuberculosis, or tuberculosis chemoprophylaxis (see Appendix). A positive tuberculin test was defined as an induration of $5 \mathrm{~mm}$ or greater (transverse diameter to $0.1 \mathrm{ml}$ of 1:1000 tuberculin PPD solution by intradermal injection read at 48-72 hours) for a Mantoux test, or a grade 2 reaction or greater (read at 7 days) for a Heaf test. ${ }^{11}$

Appropriateness of management was assessed by the following criteria based on the British Thoracic Society recommendations ${ }^{10}$ : prompt case notification; joint management by a paediatrician and chest doctor; and treatment with a recommended chemotherapy or chemoprophylaxis regimen (table 1).

\section{Results}

EPIDEMIOLOGY AND CLINICAL FEATURES

Sixty three patients were identified, of whom 48 met the case definition for tuberculosis (eight confirmed, 34 probable, and six possi-

Table 2 Demographic characteristics and clinical features of 48 patients with childhood tuberculosis

\begin{tabular}{|c|c|}
\hline Characteristic & No (\%) \\
\hline Male & $29(60)$ \\
\hline \multicolumn{2}{|l|}{ Age (years) } \\
\hline $0-4$ & $19(40)$ \\
\hline $5-9$ & $18(38)$ \\
\hline $10-14$ & $11(23)$ \\
\hline \multicolumn{2}{|l|}{ Ethnic origin } \\
\hline White & $38(79)$ \\
\hline Indian subcontinent & $7(15)$ \\
\hline Afro-Caribbean & $0(0)$ \\
\hline Other & $3(6)$ \\
\hline \multicolumn{2}{|l|}{ Site of disease } \\
\hline Pulmonary & $31(65)$ \\
\hline Intrathoracic lymph nodes & $3(6)$ \\
\hline Extrathoracic lymph nodes & $6(13)$ \\
\hline Central nervous system & $2(4)$ \\
\hline Bone and joint & $1(2)$ \\
\hline Testicular & $1(2)$ \\
\hline Not known & $4(8)$ \\
\hline \multicolumn{2}{|l|}{ Chest radiograph } \\
\hline Normal & $13(27)$ \\
\hline Cavitary tuberculosis & $1(2)$ \\
\hline Non-cavitary consistent with tuberculosis & $21(44)$ \\
\hline Non-cavitary unusual for tuberculosis & $3(6)$ \\
\hline Other abnormality & $10(21)$ \\
\hline \multicolumn{2}{|l|}{ Microbiological confirmation } \\
\hline Culture positive & $8(17)$ \\
\hline Culture negative & $16(33)$ \\
\hline Culture not done & $24(50)$ \\
\hline \multicolumn{2}{|l|}{ Tuberculin test } \\
\hline Mantoux or Heaf positive & $36(75)$ \\
\hline Mantoux or Heaf negative & $6(13)$ \\
\hline Result not recorded & $2(4)$ \\
\hline Not done & $4(8)$ \\
\hline \multicolumn{2}{|l|}{ BCG immunisation status } \\
\hline Had BCG & $6(13)$ \\
\hline Not had BCG & $30(63)$ \\
\hline Not recorded & $12(25)$ \\
\hline
\end{tabular}

ble) and 10 for tuberculosis chemoprophylaxis. Of the remainder, three had opportunist mycobacterial infection and two had insufficient evidence to substantiate a diagnosis of tuberculosis. Thirty one patients presented with symptoms, but the remaining $17(35 \%)$ patients were detected by tuberculosis contact screening. None of the patients with tuberculosis was detected as a result of the routine school BCG immunisation programme, although one 13 year old boy (whose father was later found to have pulmonary tuberculosis) was referred by the school programme for tuberculosis chemoprophylaxis.

The mean annual tuberculosis incidence was $1.2 / 100000$, with a peak annual incidence of $1.7 / 100000$ in 1991 and 1992 . At the time of the 1991 census, Wales had a population of 2.9 million, of whom 193000 were aged 0-4 years, 187000 were aged 5-9 years, and 178000 were aged 10-14 years. Of these, $97.5 \%$ were white, $1 \%$ originated from the Indian subcontinent, $0.1 \%$ were Afro-Caribbean, and $1.4 \%$ were of other ethnic origins (black African, black other, Chinese, other Asian). Table 2 gives the demographic characteristics and clinical presentations of the patients.

Pulmonary disease was the most common presentation. Of the 10 patients examined for acid fast bacilli by sputum smear, however, only two were positive. Other specimens examined bacteriologically included gastric aspirates (four), lymph nodes (six), early morning urine (three), cough swab (one), and cerebrospinal fluid (three). Of these, six were positive on culture. In 24 patients no culture was performed. Tuberculin testing was performed in $44(92 \%)$ of the 48 patients. Twenty four (50\%) patients had a Mantoux test and $22(46 \%)$ had a Heaf test, including two patients who had both tests.

Documentation of the tuberculin test results was poor. The strength of tuberculin used was recorded for only nine Mantoux tests, and the diameter of induration was precisely measured in only seven of the 19 positive Mantoux tests, the remaining results being reported as "weakly" or "strongly" positive. The grade of reaction was recorded in only 12 of the 19 positive Heaf tests. BCG immunisation status was not recorded in $25 \%$ of patients. Two $(20 \%)$ patients of ethnic minority origin and four $(11 \%)$ white patients had been immunised with BCG, the latter after contact with patients with tuberculosis.

PATIENT MANAGEMENT

The patients were mostly managed by paediatricians alone $(69 \%)$ or chest doctors alone $(25 \%)$, but rarely by both (6\%). Paediatricians were more likely to undertake microbiological investigations (17 of 33 (52\%) patients) and to perform Mantoux tests (19 of $33(58 \%)$ patients) than chest doctors (one of $12(8 \%)$ patients and four of $12(33 \%)$ patients, respectively). A similar proportion of symptomatic patients $(73 \%)$ and patients detected by contact tracing $(67 \%)$ were managed by paediatricians. Seventeen (35\%) patients with tuberculosis were not notified, but there was no difference in the proportion notified by paedia- 
Table 3 Appropriateness of chemotherapy regimens used for 48 patients with childhood tuberculosis according to site of disease

\begin{tabular}{lll}
\hline Site of disease & Chemotherapy regimen & $\begin{array}{l}\text { No (\%) of } \\
\text { patients }\end{array}$ \\
\hline Pulmonary & $\begin{array}{l}\text { British Thoracic Society } \\
\text { recommended regimen } \\
\text { Over treated }\end{array}$ & $5(10)$ \\
& $\begin{array}{l}\text { Under treated } \\
\text { Non-pulmonary }\end{array}$ & $11(23)$ \\
& $\begin{array}{l}\text { Lost to follow up } \\
\text { British Thoracic Society } \\
\text { recommended regimen }\end{array}$ & $9(13)$ \\
& $\begin{array}{l}\text { Over treated } \\
\text { Under treated } \dagger\end{array}$ & $4(8)$ \\
& Lost to follow up & $8(17)$ \\
Not known & British Thoracic Society \\
& $\quad$ recommended regimen & 0 \\
& $\begin{array}{l}\text { Over treated } \\
\text { Under treated }\end{array}$ & 0 \\
& Lost to follow up & $4(8)$ \\
\end{tabular}

*Too many drugs or excessive duration of treatment.

†Too few drugs or inadequate duration of treatment.

tricians or chest doctors. Similarly, the likelihood of notification was not influenced by the source of the patient (acute illness or contact tracing), the site of disease, or the geographical area.

Only five $(10 \%)$ patients were managed according to British Thoracic Society guidelines. All of these were patients with pulmonary tuberculosis treated with a two drug (rifampicin and isoniazid) regimen (table 3 ). Eighteen (38\%) patients were undertreated either because they were given an inappropriate drug combination or because the duration of treatment was inadequate. This included 11 patients unnecessarily treated with ethambutol. Many patients were over treated; $23 \%$ of patients with pulmonary disease were treated for more than nine months and $8 \%$ of non-pulmonary patients were treated for more than six months. The longest duration of treatment was 32 months in a patient with tuberculous meningitis. Four patients were inappropriately treated with a quadruple drug regimen (two with pulmonary disease, one with lymph node disease, and one with tuberculous meningitis). Twenty five (52\%) patients received unnecessary treatment with pyridoxine. Three patients required steroids, two for tuberculous meningitis and one for phlyctenular conjunctivitis. Surgery was performed in 10 patients, mostly for the removal of lymph nodes. No death occurred. All 10 patients treated by chemoprophylaxis received two drugs (isoniazid and rifampicin); nine were over treated (more than six months of treatment) and one was under treated (two months only). No patient required a change of treatment as a result of an adverse drug reaction.

Paediatricians were less likely than chest doctors to under treat patients with tuberculosis (eight of $33 v$ nine of 12; odds ratio 0.11 ( $95 \%$ confidence interval 0.02 to $0.59, \mathrm{p}<$ $0.01)$ ), although nine of the 10 patients lost to follow up were under the care of a paediatrician. There was no significant difference with respect to the proportion of over treated patients or the inappropriate use of ethambutol.

\section{Key messages}

- One third of patients with childhood tuberculosis were not notified

- Most case of tuberculosis were in white children

- Two thirds of patients had pulmonary disease; only two had tuberculous meningitis and none died

- BCG immunisation status and tuberculin skin test results were poorly documented

- Very few patients were jointly managed by paediatricians and chest doctors

- Drug treatment was often inappropriate, either with respect to the choice of drugs or the duration of treatment

\section{Discussion}

This study found that the management of children with tuberculosis in Wales falls short of the recommendations of the British Thoracic Society. ${ }^{10}$ There was an undernotification of cases, infrequent shared care between paediatricians and chest doctors, and inappropriate treatment, both with respect to the choice of drugs and the duration of treatment. Childhood tuberculosis remains uncommon in Wales. There is evidence, however, of an increase in childhood tuberculosis, particularly in urban areas and among new immigrants and refugees. ${ }^{45}$ This, in turn, implies a high rate of recent transmission of tuberculosis. Diagnostic awareness and familiarity with recommendations on the management of tuberculosis in children are therefore particularly important.

Undernotification of tuberculosis is well recognised and estimated to be around $20-30 \% .^{12}{ }^{13}$ In this study, there was a $35 \%$ undernotification of tuberculosis in children. Childhood tuberculosis is almost invariably the result of recent infection from an adult with tuberculosis. Undernotification in children thus has significant implications for tuberculosis control as it may lead to an infective case of tuberculosis in an adult remaining unrecognised. Many cases of childhood tuberculosis could probably be prevented if case detection of adults and subsequent contact tracing were carried out more promptly and efficiently. ${ }^{14} 15$

The study also found that less than a quarter of patients with tuberculosis from ethnic minority groups had been immunised with BCG, indicating further lost opportunities for prevention. As far as we could ascertain, the primary failure was with the neonatal BCG programme and not with the immigration screening procedure. Similarly poor rates of BCG immunisation have been noted in other studies of children with tuberculosis. ${ }^{816}{ }^{17} \mathrm{Neo}-$ natal BCG immunisation is recommended in the UK for all infants born to immigrants from countries with a high prevalence of tuberculosis. ${ }^{11}$ BCG immunisation coverage is not routinely monitored in the $\mathrm{UK}$, however, unlike the remainder of the childhood immunisation programme, and at least one study has shown considerable room for improvement. ${ }^{18} 19$ 
The tuberculin skin test is an important diagnostic test for tuberculosis in children. Our study found that tuberculin tests were often not properly conducted (the Mantoux test reaction was only accurately measured in $40 \%$ of tests) or the results were not clearly recorded. Isolation of mycobacteria from children is difficult as they often do not produce sputum and the sensitivity of diagnostic tests is low because the number of organisms that cause the disease in children is generally much lower than in adults. Nevertheless, it is desirable to seek culture confirmation of tuberculosis if at all possible. Attempts at isolating mycobacteria were made in only half of the patients in this study, of whom a third were culture positive.

Although there is little research on tuberculosis chemotherapy in children, the same basic principles as adults are considered to apply. There is, in fact, considerable consensus on the treatment of childhood tuberculosis and British ${ }^{10}$ American, ${ }^{20}{ }^{21}$ and Canadian ${ }^{22}$ recommendations on chemotherapy for pulmonary tuberculosis in children are now identical. In this study, one third of the patients were overtreated, most commonly with regard to the duration of treatment or the number of drugs against tuberculosis used. None of the chest doctors used the triple drug regimen of isoniazid, rifampicin, and pyrazinamide. Thirteen patients received ethambutol, although this is only recommended in exceptional circumstances (table 1) because of the difficulties of detecting ocular side effects in children. Pyridoxine (to prevent the peripheral neuropathy associated with isoniazid) was also used in many patients, although this is only routinely recommended for patients at high risk such as those with diabetes, alcoholics, and those with chronic renal failure. ${ }^{10}$

No published British guidelines on the management of childhood tuberculosis were available before 1990. It is possible that the issue of guidelines may have led to an improvement in practice during the second half of our study. The findings suggest, however, that the British Thoracic Society recommendations on the management of childhood tuberculosis should be more widely known, particularly among paediatricians. To monitor and improve the management of childhood tuberculosis in Wales, we have disseminated the results of this study widely among paediatricians, chest doctors, and specialist tuberculosis nurses. New British Thoracic Society guidelines have been published on the control and prevention of tuberculosis ${ }^{23}$ and on notification. ${ }^{24}$ Guidelines for treating children will be published soon. Tuberculosis is now included in the Welsh paediatric surveillance scheme, which is based on monthly returns on a small range of uncommon childhood disorders made by all paediatricians, and a similar scheme has been established with chest doctors. This will enable us to identify any improvements in current practice that occur as a result.

Shared care between paediatricians and chest doctors should ensure that adequate support is available from the full chest clinic team throughout the duration of the child's treat- ment. This would help overcome many of the deficiencies identified by our study and should improve the management of tuberculosis in children.

We thank Mrs J Jones, audit coordinator, Llandough Hospital for help with the collection of data, and Miss $\mathrm{H}$ James for the preparation of the manuscript.

1 Medical Research Council Tuberculosis and Chest Diseases Unit. Tuberculosis in children in a national survey of notifications in England and Wales, 1978-9. Arch Dis Child 1982;57:734-41.

2 Medical Research Council Tuberculosis and Chest Diseases Unit. Tuberculosis in children: a national survey of notifications in England and Wales in 1983. Arch Dis Child 1988;63:266-76.

3 Medical Research Council Cardiothoracic Epidemiology Group. Tuberculosis in children: a national survey of notifroup. Tuberculosis in children: a national survey of notifications in England

4 Hayward AC, Watson JM. Tuberculosis in England and Wales 1982-1993: notifications exceed predictions. Commun Dis Rep CDR Rev 1995;5:R29-33.

5 Bhatti N, Law MR, Morris JK, Halliday R, Moore-Gillon J. Increasing incidence of tuberculosis in England and Wales: a study of the likely causes. BMF 1995;310:967-9.

6 Goodyear HM, Moore-Gillon JC, Price EH, Larcher VF, Savage MO, Wood CBS. Mycobacterial infection in an inner-city children's hospital. Arch Dis Child 1993;69:22931

7 Cantwell M, Snider D Jr, Cauthen G, Onorato I. Epidemiology of tuberculosis in the United States, 1985 through 1992. fAMA 1994;272:535-9.

8 Starke JR, Jacobs RF, Jereb J. Resurgence of tuberculosis in children. F Pediatr 1992;120:839-55.

9 Medical Research Council Tuberculosis and Chest Diseases Unit. Management and outcome of chemotherapy for childhood tuberculosis. Arch Dis Child 1989;64:1004-12.

10 Subcommittee of the Joint Tuberculosis Committee. Chemotherapy and management of tuberculosis in the United Kingdom: recommendations of the Joint Tuberculosis Committee of the British Thoracic Society. Thorax 1990;45:403-8.

11 United Kingdom Health Departments. Joint Committee on Vaccination and Immunisation. Immunisation against infectious disease. London: HMSO, 1992.

12 Sheldon CD, King K, Cock H, Wilkinson P, Barnes NC. Notification of tuberculosis: how many cases are never reported? Thorax 1992;47:1015-18.

13 Roderick PJ, Connelly JB. The problems of monitoring tuberculosis in an inner-city health district: integrated information is required. Public Health 1992;106:193-201.

Nolan R. Childhood tuberculosis in North Carolina: a study of the opportunities for intervention in the transmission of
tuberculosis in children. Am $\mathcal{F}$ Public Health 1986;76:2630.

15 Driver C, Luallen J, Good W, Valway S, Frieden T, Onorato I. Tuberculosis in children younger than five years old: New York City. Pediatr Infect Dis f 1995;14:112-17.

16 Cundall DB, Pearson SB. Inner city tuberculosis and immunisation policy. Arch Dis Child 1988;63:964-6.

7 Goldman JM, Teale C, Cundall DB, Pearson SB. Childhood tuberculosis in Leeds, 1982-90: social and ethnic factors and the role of the contact clinic in diagnosis. Thorax 1994; 49:184-5.

18 Evans MR. Is tuberculosis taken seriously in the United Kingdom? BMF 1995;311:1483-5.

19 Ahmed S, Hicks NR, Stanwell-Smith R. Policy and practice-an audit of neonatal BCG immunisation in Avon. F Public Health Med 1992;14:389-92.

20 American Academy of Pediatrics Committee on Infectious Disease. Chemotherapy for tuberculosis in infants and Disease. Chemotherapy for tubercul
children. Pediatrics 1992;89:161-5.

21 American Thoracic Society and Center for Disease Control and Prevention. Treatment of tuberculosis and tuberculosis infection in adults and children. Am $\mathcal{F}$ Respir Crit Care Med 1994;149:1359-74.

22 Canadian Paediatric Society Infectious Disease and Immunization Committee. Short-course therapy for tuberculosis in infants and children. Can Med Assoc F 1994;150:1233-9.

23 Joint Tuberculosis Committee of the British Thoracic Society. Control and prevention of tuberculosis in the United Kingdom: code of practice 1994. Thorax 1994;49:1193200 .

24 Joint Tuberculosis Committee of the British Thoracic Society. Notification of tuberculosis: an updated code of practice for England and Wales. $\mathcal{f} R$ Coll Physicians Lond 1997;31:299-303.

\section{Appendix}

Case definitions are given in this section for tuberculosis and tuberculosis chemoprophylaxis. 
CONFIRMED CASE

Culture confirmed disease due to Mycobacterium tuberculosis complex infection ( $M$ tuberculosis, $M$ bovis, $M$ africanum).

PROBABLE CASE

In the absence of culture confirmation: (a) signs or symptoms, or both, compatible with tuberculosis; and (b) response to treatment with two or more drugs against tuberculosis (at least two of the drugs listed in the British National Formulary section on anti-tuberculous drugs (5.1.9)); and (c) either microscopic/ histological evidence of mycobacterial infection (identification of acid fast bacilli in a clinical specimen such as sputum or the presence of granuloma or caseation, or both, with or without acid fast bacilli, on histological section) or positive tuberculin test result (Mantoux-5 $\mathrm{mm}$ or greater induration (transverse diameter to $0.1 \mathrm{ml}$ of $1: 1000$ tuberculin PPD solution) by intradermal injection read at $48-72$ hours; Heaf-grade 2 or greater reaction to test read at 7 days).
POSSIBLE CASE

In the absence of culture confirmation, microscopic/histological evidence of mycobacterial infection, or positive tuberculin test result: (a) signs or symptoms, or both, compatible with tuberculosis; and (b) response to treatment with two or more drugs against tuberculosis (at least two of the drugs listed in the British National Formulary section on antituberculous drugs (5.1.9)).

TUBERCULOSIS CHEMOPROPHYLAXIS

Contacts who have a strongly positive Mantoux or Heaf test reaction, but no clinical or radiological evidence of tuberculosis disease, such as: (a) those under the age of 16 years who have had BCG and who have a positive Mantoux test greater than $5 \mathrm{~mm}$ or Heaf test grade $2-4$; (b) those in whom recent tuberculin conversion has been noted; and (c) those less than 2 years of age who are close contacts of patients with positive smears and who have not had BCG, irrespective of their tuberculin status. 